\title{
Características produtivas de Kinosternon scorpioides nas fases de acasalamento, postura e eclosão, criados em cativeiro na Amazônia
}

Costa, J.S. ${ }^{1 @ ;}$ Marques, L.C. ${ }^{2}$; Matos, A.S. ${ }^{1}$; Silva, C.S. ${ }^{1}$; Figueiró, M.R. ${ }^{3}$; Sales, R.L. ${ }^{3}$; Da Silva Filho, E. ${ }^{4}$; Guimarães, D.A.A. ${ }^{5}$ e Marques, J.R.F. ${ }^{3}$

'Programa de Pós-Graduação em Ciência Animal. Universidade Federal do Pará (UFPA). Campus Belém. Pará. Brasil.

2Universidade da Amazônia (UNAMA). Belém. Pará. Brasil.

${ }^{3}$ Embrapa Amazônia Oriental. Belém. Pará. Brasil.

${ }^{4}$ Universidade Federal Rural da Amazônia (UFRA). Campus Belém. Pará. Brasil.

5Universidade Federal do Pará (UFPA). Campus Belém. Pará. Brasil.

PALAVRAS-CHAVE ADICIONAIS

Ácido graxo volátil.

Coproduto.

Glicose.

Rúmen.

\section{RESUMO}

Objetivou-se estudar os índices das características produtivas de muçuãs (Kinosternon scorpioides) visando nortear manejos adequados para a sustentabilidade da atividade. Analisou-se 84 acasalamentos, 742 posturas e 1979 nascimentos em cativeiro, determinando-se: a estação reprodutiva e a distribuição dos acasalamentos; a duração do acasalamento; a razão sexual; a duração do período de desenvolvimento do ovo (PDO); a frequência de múltiplas posturas; duração do período de nidificação; a correlação entre tempo de nidificação e número de ovos; o número de ovos viáveis; a correlação entre número, peso, comprimento e largura dos ovos; a análise descritiva para as características biométricas e peso dos ovos; a duração do período de incubação; a estação de nascimentos; as análises descritivas de peso e medidas corporais para os filhotes e a correlação entre as variáveis. A estação reprodutiva é sazonal, concentrando os acasalamentos na época mais chuvosa do ano com duração média de 00:23:00 h, com razão sexual de 2,38 $\pm 1,67: 1$. O PDO foi de 122,98 \pm 45,38 dias. A distribuição das posturas apresentou uma tendência de clímax entre junho e agosto. A duração da nidificação foi de 02:57:00 h. A viabilidade dos ovos foi de 20\%. A correlação entre as características biométricas, peso e o número de ovos foi positiva. Os resultados biométricos do ovo foram de $34,5 \mathrm{~mm}$ de comprimento e $18 \mathrm{~mm}$ de largura, com peso de 7,90 g. O período de incubação foi de 129,31 $\pm 19,57$ dias, com os nascimentos se concentrando entre os meses de setembro a dezembro. As correlações das características biométricas com o peso dos filhotes foram positivas. substituição ao milho no concentrado de bezerros até 60 dias de idade.

\section{Productive characteristics of Kinosternon scorpioides, during copulation, ovarian activity, and hatching phases, in captivity in Amazon}

\section{SUMMARY}

\section{ADDITIONAL KEYWORDS}

Byproduct.

Glucose.

Rumen.

Volatile fatty acid.

\section{INFORMATION}

Cronología del artículo.

Recibido/Received: 26.04.2016

Aceptado/Accepted: 17.02.2017

On-line: 15.07 .2017

Correspondencia a los autores/Contact e-mail:

juliane_silvacosta18@yahoo.com.br
The aim of this article was to study the indexes of muçuã)(Kinosternon scorpioides) productive characteristics during copulation, egg laying and hatching, in order to provide an adequate management for the sustainability of the activity. 84 couplings, 742 egg laying and 1,979 births in captivity were analyzed, from which we determined the reproductive season and distribution of couplings; coupling duration; the sexual reason; duration of the egg development period (EDP), frequency of multiple egg laying; duration of nesting period; correlation between nesting time and the number of eggs; the number of viable eggs; the correlation between egg number, weight, length and the descriptive analysis for biometric characteristics and egg weight; duration of the incubation period; the season for births; descriptive analyses of hatchling weight, body measurements and the correlation between variables. The reproductive period is seasonal, concentrating couplings during the rainy season, and the average coupling duration was $00: 23: 00 \mathrm{~h}$, with sexual reason of 2.38 $\pm 1.67: 1$. The EPD was $122.98 \pm 45.38$ days. Nesting distribution showed a climax tendency between the of June and August. The nesting average was $02: 57: 00 \mathrm{~h}$. The egg viability was $20 \%$. The correlation between biometric characteristics, weight, and the number of eggs laid were positive. The egg biometric results were $34.5 \mathrm{~mm}$ long and $18 \mathrm{~mm}$ wide, with weight of $7.90 \mathrm{~g}$. The incubation period was $129.31 \pm 19.57$ days, with births concentrating between the months of September and December. All correlations of biometric characteristics with hatching weight were positive.

\section{INTRODUÇÃO}

O quelônio conhecido regionalmente por muçuã (Kinosternon scorpioides) é um dos animais mais preda- dos da região da ilha de Marajó, por ser um alimento muito apreciado na culinária local, no campo e nas cidades, causando baixas consideráveis no seu estoque natural. 
A caça predatória exerce uma pressão muito forte sobre as populações naturais das espécies de interesse comercial, proporcionando seu declínio e comprometendo o funcionamento do ecossistema onde estão inseridas, bem como das comunidades e economias locais que dependem destas espécies (Redford, 1997; Clayton e Milner-Gulland, 2000; Sirvinskas, 2002; Traffic, 2005).

Apesar da ocorrência da caça predatória e comércio ilegal de espécies silvestres, a fauna brasileira está protegida pela Lei de Proteção à Fauna (Lei n ${ }^{\circ}$ 5.197, de 03 de janeiro de 1967) que incentiva a criação racional em cativeiro de algumas espécies de animais silvestres, contudo, juntamente com a Lei de Crimes Ambientais (Lei n ${ }^{\circ}$ 9.605, de 12 de fevereiro de 1998) proíbe a caça, transporte e comercialização de animais silvestres no país.

Na Amazônia há grande pressão predatória sobre mamíferos e repteis. Dentre os repteis a maior pressão predatória é sobre os quelônios (Sampaio, 2003; Araújo, 2009). Diferentemente do ambiente natural o estudo em cativeiro tem sido de extrema importância, uma vez que possibilita a avaliação mais detalhada dos padrões comportamentais reprodutivos (Carpenter, 1980; Molina, 1989; Carpenter; Ferguson, 1997; Schneider et al., 2010; Silva, 2011).

Portanto, o maior conhecimento sobre a biologia de espécies altamente predadas e o seu comportamento reprodutivo é de grande valia para o direcionamento de ações conservacionistas da fauna de vide livre e para o manejo adequado em cativeiro. Uma vez que o conhecimento científico sobre a reprodução de quelônios sul-americanos ainda é limitado.

Objetivou-se estudar os índices de características reprodutivas nas fases de acasalamento, postura e eclosão, em sistema de criação em cativeiro, visando nortear manejos adequados para a maior sustentabilidade da atividade.

\section{MATERIAL E MÉTODOS}

O banco de dados utilizado neste trabalho é proveniente da Coleção Biológica de muçuãs da Embrapa registrada pela Secretaria de Estado de Meio Ambiente do Pará, conforme a Licença de Operações $\mathrm{N}^{\mathrm{o}}$ 7310/2014, como Criadouro científico de fauna silvestre para fins de pesquisa da Embrapa Amazônia Oriental, localizado no município de Salvaterra, coordenadas $48^{\circ} 30^{\prime} 54^{\prime \prime}$ de longitude $W$ e $00^{\circ} 45^{\prime} 21^{\prime \prime}$ de latitude $S$, na ilha de Marajó, Estado do Pará, Brasil. A área total do criatório é de $200 \mathrm{~m}^{2}$, dividida em cinco ambientes, conforme as fases de desenvolvimento zootécnico dos animais, ou seja: incubatório artificial $\left(6 \mathrm{~m}^{2}\right)$, Berçário (6 $\mathrm{m}^{2}$ ) - animais até $50 \mathrm{~g}$, contendo 441 indivíduos; Recria $\left(30 \mathrm{~m}^{2}\right)$ - animais acima de $51 \mathrm{~g}$ até $250 \mathrm{~g}$, contendo 69 indivíduos; Reprodução $\left(50 \mathrm{~m}^{2}\right)$ - animais acima de 251 $\mathrm{g}$, contendo 632 indivíduos, destes 67 machos e, um quarentenário $\left(8 \mathrm{~m}^{2}\right)$, construído conforme preceitua a Instrução Normativa N 169 do IBAMA/2008. Ressalta-se que o muçuã se apresenta com o comportamento claramente promíscuo, permitindo a aproximação de pessoas mesmo quando está em situações de acasalamento, alimentação, postura, etc., permitindo a apro- ximação e manipulação sem alterações na reação. $\mathrm{O}$ ambiente estava dividido em $70 \%$ de terra firme, com areia clara, pouco orgânica, e 30\% de água corrente, com uma lâmina em torno dos $20 \mathrm{~cm}$ de altura.

A classificação climática, segundo Koppen (1948) apresenta tipo climático Ami, com estação menos chuvosa (julho a dezembro) e mais chuvosa (janeiro a junho), com temperatura média de $27^{\circ} \mathrm{C}$, precipitação média anual de $2943 \mathrm{~mm}$ e umidade relativa do ar 85\%.

A pesquisa foi submetida e aprovada pela Comissão de Ética no Uso de Animais (CEUA) da Embrapa Amazônia Oriental, Belém-PA, Nº de protocolo 001/2016. A mesma foi conduzida no período de 2007 a 2015, em que foram analisados 84 acasalamentos, 742 posturas e 1.979 nascimentos, relacionados ao desempenho reprodutivo dos animais em cativeiro. Para as análises reprodutivas, foram avaliados acasalamentos (entre os anos de 2008 a 2015), o comportamento de nidificação e posturas (entre os anos de 2007 a 2015), e incubação (entre os anos de 2007 a 2015).

Todas as observações realizadas neste estudo foram possíveis mediante identificação individual dos animais com o uso de microchip da marca Partners $\AA$, que é um microcircuito eletrônico medindo $12 \times 2 \mathrm{~mm}$, implantado no lado esquerdo, na região cervical, abaixo da carapaça. Este tipo de identificação permitiu a observação e identificação dos animais nos diferentes eventos reprodutivos (acasalamento, postura e ninhos em eclosão).

Para as características avaliadas determinou-se: a estação de acasalamento (contínua ou sazonal) e a distribuição dos acasalamentos; a duração do acasalamento; a relação macho:fêmea; a duração do período de desenvolvimento do ovo (PDO); a frequência de múltiplas posturas por estação reprodutiva; duração do período de nidificação; a correlação entre tempo de nidificação e número de ovos; o número de ovos viáveis por período reprodutivo; a correlação entre número, peso, comprimento e largura dos ovos (figura 1); a análise descritiva para as características biométricas

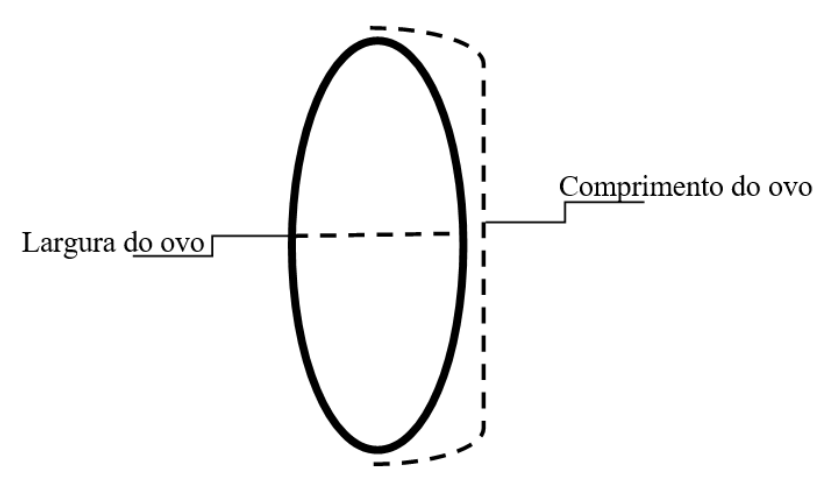

Figura 1. Esquematização da metodologia padrão para morfometria dos ovos de muçuãs (Kinosternon scorpioides) mantidos no criatório cientifico da Embrapa Amazônia Oriental - PA (Schematics of standard methodology for morphometric of eggs of muçuãs (Kinosternon scorpioides) kept in the scientific breeding centre of Embrapa Amazônia Oriental - PA). 
e peso dos ovos; a duração do período de incubação; a estação de nascimentos; as análises descritivas de peso e medidas corporais para os filhotes e a correlação das variáveis.

Na análise de duração do PDO (intervalo de tempo que vai do acasalamento à postura), em dias, organizou-se em três grupos de acordo com o número de dias: Grupo 1 = 1 a 100 dias; Grupo 2 = 101 a 150 dias; Grupo 3=mais de 151 dias.

Para a análise da duração do período de nidificação considerou-se os seguintes comportamentos: saída da fêmea da água, abertura do ninho, postura dos ovos, fechamento e abandono do ninho. Todos os comportamentos utilizados neste estudo basearam-se nas anotações referentes a observações diárias dos funcionários do criatório, em tempo real.

Nas análises envolvendo as medidas morfométricas do ovo, utilizou-se a seguinte metodologia (Figura 1): comprimento e largura retilínea do ovo, em milímetros, com o auxílio de paquímetro padrão de precisão, além de observações relevantes com relação às posturas e aos aspectos dos ovos, através de medidas periódicas, nas diferentes épocas do ano (chuvosa e menos chuvosa).

Para as análises envolvendo as medidas morfométricas dos filhotes utilizou-se a seguinte metodologia: comprimento retilíneo da carapaça - CC, largura retilínea da carapaça - LC, comprimento retilíneo do plas-

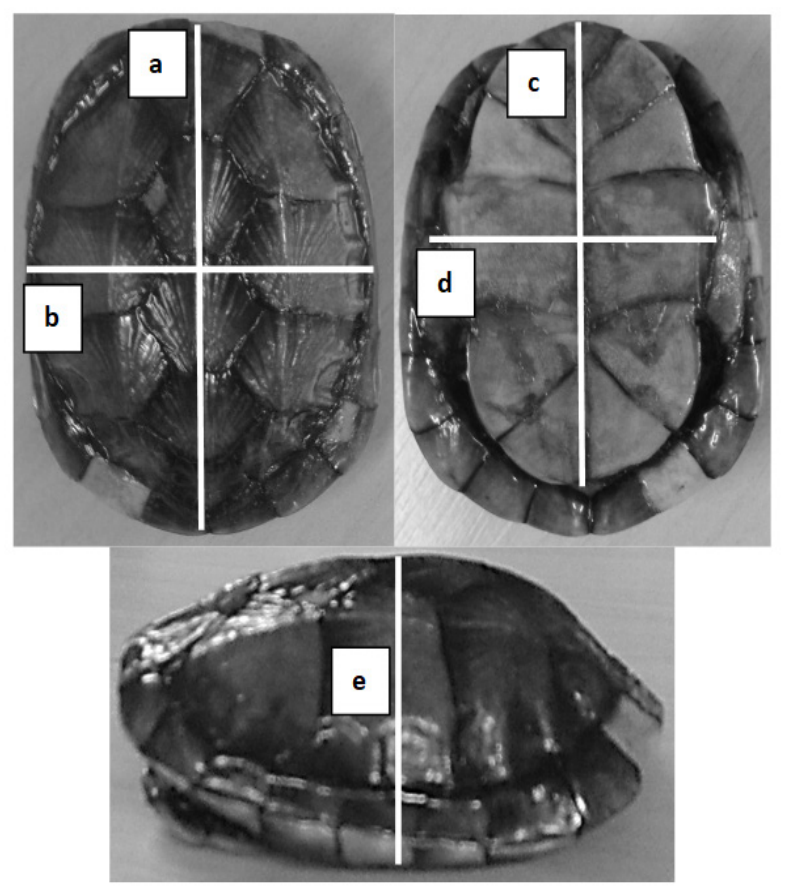

Figura 2. Esquematização da metodologia padrão para morfometria dos filhotes: (a) comprimento retilíneo da carapaça - CC; (b) largura retilínea da carapaça - LC; (c) comprimento retilíneo do plastrão - CP; (d) largura retilínea do plastrão - LP; (e) altura - ALT (Schematics of standard methodology for morphometry of chicks: (a) straight carapace length - CC; (b) straight carapace width - LC; (c) rectilinear length of the plastron - LC; (d) rectilinear of the width plastron - LP; (f) height - ALT). trão - CP, largura retilínea do plastrão - LP e a altura - ALT, do plastrão ao ponto mais alto da carapaça, em milímetros, com o auxílio de paquímetro digital, marca Worker $^{\circledR}$, padrão de precisão de 0,01 mm (figura 2).

Para todas as análises envolvendo a variável peso, seja dos ovos ou dos filhotes, utilizou-se para as pesagens balança semianalítica de precisão eletrônica 0,001g, marca Gehaka ${ }^{\circledR}$ modelo BG 8000.

Para a análise de peso dos filhotes, organizou-se em quatro grupos: Grupo 1=menos que 2 g; Grupo $2=$ 2,1 a 4 g; Grupo $3=4,1$ a 6 g; Grupo 4 = maior que 6 g.

O banco de dados foi organizado e padronizado em planilhas do software Excel, Microsoft Office ${ }^{\circledR} 2010$, contendo colunas específicas para cada categoria reprodutiva. Para a categoria de acasalamento utilizouse a planilha contendo: ano, data de acasalamento, identificação da fêmea e do macho, inicio, fim e duração individual de cada acasalamento em hh:mm e observações gerais. Para a categoria de postura utilizouse a planilha contendo: identificação da fêmea, data da postura, identificação do ninho, inicio, fim e duração individual de cada postura em hh:mm, número de ovos por fêmea e/ou ninho, peso e biometria do ovo (comprimento e largura) e observações gerais. E para a categoria de eclosão utilizou-se a planilha contendo: identificação da fêmea, data da eclosão, identificação do ninho, identificação do ovo, identificação do filhote, peso e biometria do filhote (comprimento da carapaça, comprimento do plastrão, largura da carapaça, largura do plastrão e altura) e observações gerais.

Para a realização das análises estatísticas das características, que se apresentam com distribuição normal, utilizou-se o programa estatístico SAS (2004) for Windows (Statistical Analysis System, v. 8.02) onde realizou-se os procedimentos MEANS/ FREQ/ ANOVA e Correlação de Pearson $(p \leq 0,0001)$. Para as análises comparativas de médias utilizou-se o teste de Duncan $(\mathrm{p}<0,001)$, entendendo-se que tais análises satisfaziam o escopo dos estudos, sendo que os aspectos importantes para a interpretação das análises era a detecção das diversas influências sobre as características, inclusive as correlações entre elas. Respeitou-se, ainda, os níveis de significâncias estabelecidos pelos programas utilizados. Os gráficos foram plotados no software Excel.

\section{RESULTADOS E DISCUSSÃO}

As observações de 84 acasalamentos registrados no período de 2008 a 2015 constataram que o período reprodutivo observado foi sazonal, concentrando-se na época mais chuvosa do ano na ilha de Marajó, que vai de janeiro a junho, contemplando $96 \%$ dos acasalamentos registrados. Os acasalamentos iniciam no final do mês de dezembro, com tendência de pico entre o final de março e o inicio de abril. Justifica-se esta sazonalidade reprodutiva pelas características ambientais relacionadas, principalmente à nutrição, pois há maior oferta de alimentos na natureza, como também a época de clima mais ameno, influenciando a biologia reprodutiva dos animais, sendo o acasalamento claramente dependente de fatores ambientais, destacando-se a temperatura e a umidade relativa, conforme relata- 
ram Pritchard (1979) e Ferri (2002) quando afirmaram que os fatores extrínsecos determinam as atividades reprodutivas de quelônios, fazendo com que estes se reproduzam em períodos sazonais. Do mesmo modo que Souza (2004) destaca os índices pluviométricos, temperatura e umidade, como os principais fatores climáticos que moldam o comportamento reprodutivo dos quelônios. Castro (2006b) menciona a importância dos fatores ambientais para a reprodução de Kinosternon scorpioides, acrescentando que a cópula ocorre sempre dentro d'água. Entretanto, Carvalho et al. (2010) ao descrever o ritual de cópula de muçuãs relata haver indícios que o acasalamento pode ocorrer em qualquer época do ano.

Neste estudo, observou-se que a duração do acasalamento foi de $23 \mathrm{~min}$, com mínimo de dois minutos e máximo de três horas e trinta e cinco minutos, em um total de 107 observações. Nota-se uma grande variação dos tempos registrados, gerando questionamentos sobre o que pode influenciar na duração do evento. Para Souza (2004) o acasalamento de quelônios apresenta duração variável. Por outro lado, Silva (2011) em seu estudo sobre os aspectos reprodutivos do muçuã, não conseguiu determinar a duração média de acasalamento para a espécie, relatando haver grande influência do meio interno e externo do recinto, da estimulação sexual da fêmea e do comportamento agonístico entre os animais, dificultando a exatidão da determinação do tempo de cópula.

Nas observações de campo, ressalvando-se os desvios dos avaliadores, a relação macho:fêmea no recinto experimental foi de aproximadamente 1:10, sendo que as observações para os acasalamentos registrados neste estudo foi de 2,38 + 1,67: 1, com variação de um a oito fêmeas por macho (1:1 e 1:8), sendo avaliados 58 machos e 138 fêmeas envolvidas na reprodução. Tais observações são muito importantes para uma criação em cativeiro, prevendo-se uma maior eficiência reprodutiva do criadouro. A relação 1:1 foi a proporção que apresentou o maior percentual de acontecimentos observados (41,38\%), seguida da 1:2 e 1:4 com aproximadamente 26 e $12 \%$, respectivamente, demonstrando que possivelmente exista uma hierarquia social entre os animais, onde determinados machos exercem dominância sobre outros os impedindo de acasalar com as fêmeas sob seu domínio reprodutivo. De acordo com Silva (2006), em cativeiro têm-se encontrado bons resultados para a relação macho:fêmea com a proporção de um macho para três fêmeas. Silva (2011) estabeleceu uma maior proporção macho:fêmea passando de 1:3 para $1: 4$, visando reduzir os comportamentos agonísticos, também observados em seu estudo, entre os machos por disputa pelas fêmeas, pois esse tipo de comportamento é a principal causa de lesões e óbito em cativeiro. Tal afirmação concorda com Schneider et al. (2010) que afirmam que o comportamento agonístico é comum no período reprodutivo.

Para o intervalo de tempo que vai do acasalamento até a postura, em dias, ou seja, PDO, encontrou-se a média de 122,98 \pm 45,38 dias em 49 fêmeas observadas, com mínimo de três dias e máximo de 229 dias. Entende-se que a amplitude dos resultados seja em função das múltiplas cópulas (uma fêmea pode aca- salar várias vezes durante o período reprodutivo), dificultando, em alguns casos, a identificação precisa sobre qual acasalamento resultará em determinada postura, onde sugere-se resultados. Devido a frequência de múltiplas cópulas serem normais nesta espécie, fez-se um estudo de frequência de cópulas, em que dividiu-se estatisticamente em três grupos de dias de PDO: Grupo 1 (1 a 100 dias) observou-se uma frequência de 7,84\% dos eventos; no Grupo 2 (101 a 150 dias) e no Grupo 3 (mais de 151 dias) obteve-se frequência de $8,82 \%$ e $7,35 \%$ dos eventos, respectivamente.

Nas observações de 742 posturas registradas no período de 2007 a 2015 constatou- que a distribuição das posturas em cativeiro não é contínua, concentrando-se em um determinado período do ano. Pouco se sabe sobre a postura dos Kinosternídeos de vida livre, destacando a importância das observações em cativeiro. Neste estudo identificou-se período de postura sazonal para muçuãs em cativeiro, iniciando em meados do período mais chuvoso, mês de abril, estendendo-se até o período menos chuvoso da região, mês de outubro, com poucos eventos, nos dois últimos meses do ano. A distribuição das posturas apresentou uma tendência de clímax entre o final de junho e o inicio de agosto, contemplando 515 posturas das 742 registradas ao longo dos anos, corroborando com Mondolfi (1955) e Kirkpatrick (1997) que afirmam que muitas espécies de tartarugas realizam postura durante uma única estação do ano, coincidindo normalmente com temperaturas mais quentes do ar e/ou da água.

Por ser dependente de fatores ambientais (água, alimentação, temperatura e umidade) a estação de postura sofre variação conforme a localização da região estudada. Portanto, o período de postura observado na Venezuela foi entre os meses de dezembro, janeiro e fevereiro. No Estado do Maranhão, foram capturadas fêmeas ovadas durante o mês de agosto. No Zoológico de São Paulo o período de postura inicia em março e vai até o mês de abril. No Estado do Pará, município de Belém, as posturas foram entre maio e setembro, com pico entre os meses de junho e julho. Em outros estudos na ilha de Marajó-PA, as desovas ocorreram entre maio a agosto (Mondolfi, 1955; Molina, 1990; Rocha e Molina, 1990; Castro, 2006a; Rueda-Almonacid et al., 2007; Marques et al., 2008; Barreto et al., 2009; Costa et al., 2009).

A frequência de postura por fêmea em um mesmo período reprodutivo não foi significativa $(p \geq 0,0001)$, pois foram observadas fêmeas realizando apenas uma ou no máximo duas posturas no mesmo período reprodutivo, fato observado por Silva (2011) que verificou que as fêmeas podem realizar de uma a quatro posturas numa mesma estação reprodutiva e que essas têm correlação baixa e negativa com o intervalo entre posturas, justificando as múltiplas posturas com os mecanismos ecofisiológicos que é o tempo fisiológico para a formação de ovos férteis, o que pode se estender de um a quatro meses.

Neste estudo, a duração da nidificação foi de 02:57:00 \pm 01:19:00 h, com mínimo de vinte e cinco minutos e máximo de sete horas e trinta e cinco mi- 
nutos, em um total de 1077 observações. A amplitude dos tempos registrados se justifica por ter sido considerado cinco comportamentos de nidificação, os mesmos considerados por Castro (2006a) uma vez que, nem todas as fêmeas realizaram a mesma sequência de comportamentos e, quando realizam, os fazem em diferentes tempos. No comportamento de postura registrado, fêmeas foram observadas reaproveitando ninhos já feitos por outras fêmeas, dificultando a identificação do tamanho das ninhadas. Este fato foi citado por Castro (2006a) que afirmou haver uma preferência entre as fêmeas de muçuã, em aproveitar ninhos já feitos por outras fêmeas, inclusive de outras espécies de quelônios, se mantidas em mesmo ambiente.

Analisou-se a correlação entre as características de tempo de nidificação com o número de ovos por ninhada, não havendo influência significativa entre as variáveis estudadas $(p \geq 0,0001)$.

A viabilidade dos ovos estudada no período de 2007 a 2014, em percentual, consta na tabela I. Observou-se que poucos ovos incubados foram viáveis para a eclosão ao longo dos anos. Os ovos incubados artificialmente, retirados dos ninhos e levados para uma área de terra seca como incubatório visando-se minimizar a inviabilidade dos ovos por predação, principalmente, somaram 7885 no total de ovos, ao longo do período estudado, mas apenas 1975 completaram o seu desenvolvimento até a eclosão. Os anos de 2009 a 2013, foram os anos de menor viabilidade de ovos eclodidos, com média de $11 \%$, aproximada- mente, de ovos viáveis em cinco anos. As principais causas da inviabilidade dos ovos no criatório neste período foram: ovos oriundos de primíparas (>45\%), os quais eram de menor tamanho, predação por outros animais (2\%), ataque de formigas $(60 \%)$, morte embrionária (muito alta e não computada), ovo não fecundado (poucos, mas não computado), apodrecimento $(25 \%)$ e morte após eclosão (3\%).

Sobre a baixa viabilidade dos ovos incubados, Iverson (1991) em trabalho realizado com a espécie identificou que a mortalidade durante o primeiro ano de vida foi extremamente alta (81\%), em razão principalmente da predação e inviabilidade dos ovos em condições ambientais desfavoráveis. Castro (2006a) relata em seu estudo uma viabilidade de $(67,8 \%)$ de ovos que chegaram a eclodir, de 426 ovos incubados apenas 212 completaram seu desenvolvimento, destes 25 não foram encontrados e 52 filhotes não foram mensurados, restando apenas 137 animais.

Quanto às correlações entre o número de ovos e o tamanho (comprimento e largura) e peso dos ovos todas foram positivas. Sendo o peso e o comprimento do ovo as características que apresentaram as maiores correlações. Entre o peso e largura, e entre largura e comprimento foram observadas correlações medianas tabela II. Os resultados da correlação entre as características biométricas, peso e o número de ovos foram positivos, mas não significativos ( $p \geq 0,001)$, demonstrando não haver correlação entre o número de ovos por ninhada com seu tamanho e peso, conforme os estudos de Cagle (1950); Gibbons (1970); Moll e Legler

Tabela I. Percentual de viabilidade dos ovos de Kinosternon scorpioides criados em cativeiro na Amazônia Oriental, Pará, Brasil (Percentage of feasibility Kinosternon scorpioides eggs bred in captivity in eastern Amazonia, Pará, Brazil).

\begin{tabular}{lccc}
\hline Ano & $\mathrm{N}^{\circ}$ de ovos postos & $\mathrm{N}^{\circ}$ de ovos eclodidos & Viabilidade (\%) \\
\hline 2007 & 577 & 165 & 28,60 \\
2008 & 877 & 396 & 45,15 \\
2009 & 440 & 32 & 7,27 \\
2010 & 306 & 46 & 15,03 \\
2011 & 514 & 40 & 7,78 \\
2012 & 1077 & 148 & 13,74 \\
2013 & 1293 & 119 & 9,20 \\
2014 & 2801 & 1029 & 36,74 \\
\hline
\end{tabular}

Tabela II. Coeficiente de correlação de Pearson entre número de ovos, comprimento, largura e peso do ovo de muçuãs (Kinosternon scorpioides) criados no criatório da Embrapa Amazônia Oriental-PA (Pearson correlation coefficient between the number of eggs, length, width and weight of muçuãs egg (Kinosternon scorpioides) created in breeding EMBRAPA Eastern Amazon-PA).

\begin{tabular}{lllll}
\hline & $\mathrm{N}^{0}$ de ovos & Comprimento $(\mathrm{mm})$ & Largura $(\mathrm{mm})$ & Peso $(\mathrm{g})$ \\
\hline $\mathrm{N}^{\circ}$ de ovos & 1,00000 & & & \\
Comprimento $(\mathrm{mm})$ & 0,04143 & 1,00000 & & \\
& $<0,0001$ & 0,32533 & 1,00000 & \\
Largura $(\mathrm{mm})$ & 0,04446 & $<0,0001$ & & \\
& $<0,0001$ & 0,61504 & 0,36382 & 1,00000 \\
\hline
\end{tabular}

$\mathrm{N}$ (Número de observações) $=5272$ 
(1971); Tinkle et al. (1981) há variação entre as características biométricas dos ovos com o número de ovos por postura, entre populações de quelônios de diferentes regiões estudadas. Para o gênero Kinosternon observou-se que as espécies tropicais produzem ninhadas com ovos maiores, porém em menor número.

Na tabela III resume-se a análise descritiva para as características biométricas, em milímetros, e peso, em gramas, de 5272 ovos mensurados, no período de 2007 a 2015. As características biométricas médias dos ovos neste estudo foram de 34,5 mm de comprimento e de $18 \mathrm{~mm}$ de largura valores próximos aos encontrados por Castro (2006a), em que o comprimento foi de $38 \mathrm{~mm}$ e a largura de $20 \mathrm{~mm}$. O peso médio encontrado variou entre 1,60 a 13,40 g aproximando-se do valor máximo observado por Castro (2006a) de 5,70 a 13,50 g. Vogt (2008) registrou peso médio variando de 3,80 a 9,10 gramas, com peso máximo abaixo do observado neste estudo.

O tempo de incubação em animais de vida livre não é conhecido para os Kinosternídeos. Em cativeiro este tempo pode ser bastante variado. Neste estudo, a duração média do período de incubação, em dias, foi de 129,31 \pm 19,57, com mínimo de 37 dias e máxima de 172 dias, em um total de 451 registros. Resultado aproximado, para a variação máxima encontrada, foi relatado por Vogt (2008) em que o período de incubação demonstrou duração média de 176 dias. Em geral os ovos têm período de incubação, variando de três a seis meses (Kirkpatrick, 1997). No Pará, Castro (2006a) observou período de incubação variando de 111 a 164 dias, com média de 136 dias.
Os nascimentos se concentraram entre os meses de setembro a dezembro, iniciando no período considerado menos chuvoso e estendendo-se até a época mais chuvosa. Marques et al. (2008) corrobora ao registrar o inicio das eclosões no final das chuvas estendendo-se até o mês de novembro, também, na ilha de Marajó. Na Venezuela Mondolfi (1955) relatou eclosões no começo da estação chuvosa, iniciando expressivamente no mês de maio.

As características biométricas médias na fase de berçário foram de 25,92 mm de comprimento de carapaça e de 2,77 gramas de peso, demonstrado na tabela IV, tendo sido encontrados valores maiores por Barreto et al. (2009), que registraram uma média de $35+$ 0,7 mm (variação de 34,7 a 36,1 mm) de comprimento de carapaça. Marques et al. (2008) verificaram filhotes com tamanho entre 40 a $50 \mathrm{~mm}$ de comprimento de carapaça, pesando entre 6 a $7 \mathrm{~g}$. Valores mais aproximados para comprimento de carapaça foram observados no estudo de Basho e Molina (2000) em que os recém-eclodidos mediram 31,1 + 4,4 mm (variação de 28,0 a $39,1 \mathrm{~mm}$ ) de comprimento de carapaça e peso entre 5,4 a 8,2 g (média=6,53 + 1,19 g).

Na tabela $\mathbf{V}$ destacam-se as mesmas características estudadas sob a influência do peso. No estudo de influência do peso nas características estudadas observou-se que, conforme o peso dos filhotes aumenta, a média das medidas corporais também aumentam, significativamente $(\mathrm{p} \leq 0,001)$, até uma determinada amplitude de peso, após atingirem essa amplitude as medidas corporais apresentam aumento desacelerado, conforme demonstra os grupos de peso 3 e 4 que

Tabela III. Número de observações (N), média com desvio-padrão (DP), mínimo (Mín) e máximo (Máx) das características de comprimento, largura e peso de ovos de muçuãs (Kinosternon scorpioides) (Number of observations $(\mathrm{N})$, mean standard deviation (SD), minimum (Min) and maximum (Max) of the characteristics of length, width and weight muçuãs eggs (Kinosternon scorpioides)).

\begin{tabular}{lccccc}
\hline Característica & $\mathrm{N}$ & Média & DP & Mín & Máx \\
\hline Comprimento $(\mathrm{mm})$ & 5272 & 34,50 & 2,60 & 14,00 & 64,00 \\
Largura $(\mathrm{mm})$ & 5272 & 18,00 & 1,60 & 12,00 & 37,00 \\
\hline Peso $(\mathrm{g})$ & 5272 & 7,90 & 1,15 & 1,60 & 13,40 \\
\hline
\end{tabular}

Tabela IV. Número de observações (N), média com desvio-padrão (DP), mínimo (Mín) e máximo (Máx) das primeiras medições corporais e peso de muçuãs (Kinosternon scorpioides) recém-eclodidos na ilha de Marajó-PA (Number of observations $(\mathrm{N})$, mean standard deviation, minimum and maximum of the first body measurements and weight muçuãs (Kinosternon scorpioides) hatchlings on Marajó-PA island).

\begin{tabular}{lccccc}
\hline Característica & $\mathrm{N}$ & Média & DP & Mín & Máx \\
\hline Peso $(\mathrm{g})$ & 1979 & 2,77 & 5,84 & 1,00 & 4,00 \\
CC $(\mathrm{mm})$ & 1979 & 25,92 & 2,63 & 13,00 & 41,00 \\
LC $(\mathrm{mm})$ & 1979 & 16,83 & 2,24 & 5,00 & 41,00 \\
CP $(\mathrm{mm})$ & 1979 & 21,98 & 2,66 & 6,00 & 41,00 \\
LP $(\mathrm{mm})$ & 1979 & 12,79 & 2,40 & 4,00 & 41,00 \\
Alt $(\mathrm{mm})$ & 1979 & 14,57 & 1,53 & 7,00 & 41,00
\end{tabular}

CC: Comprimento da Carapaça, LC: Largura da Carapaça, CP: Comprimento do Plastrão, LP: Largura do Plastrão e Alt: Altura dos filhotes recém-eclodidos, em milímetros. 
Tabela V. Número de observações (N) e média com desvio padrão (DP) das características biométricas de Kinosternon scorpioides de acordo com o peso, entre os anos de 2007 a 2015, na ilha de Marajó-PA (Number of observations (N) and SD of the biometric characteristics of Kinosternon scorpioides according to weight, between the years 2007-2015, in Marajó-PA island).

\begin{tabular}{|c|c|c|c|c|c|c|c|c|}
\hline \multirow{2}{*}{ Característica } & \multicolumn{2}{|c|}{ Peso 1} & \multicolumn{2}{|c|}{ Peso 2} & \multicolumn{2}{|c|}{ Peso 3} & \multicolumn{2}{|c|}{ Peso 4} \\
\hline & $\mathrm{N}$ & Média_DP & $\mathrm{N}$ & Média_DP & $\mathrm{N}$ & Média_DP & $\mathrm{N}$ & Média_DP \\
\hline $\mathrm{CC}(\mathrm{mm})$ & 6 & $22,16^{c} \pm 3,87$ & 598 & $23,61^{b} \pm 1,89$ & 1218 & $26,95^{\mathrm{a}} \pm 2,10$ & 157 & $26,88^{a} \pm 2,98$ \\
\hline $\mathrm{LC}(\mathrm{mm})$ & 6 & $13,83^{c} \pm 2,92$ & 598 & $15,39^{b} \pm 1,70$ & 1218 & $17,52^{a} \pm 2,16$ & 157 & $17,14^{\mathrm{a}} \pm 1,89$ \\
\hline $\mathrm{CP}(\mathrm{mm})$ & 6 & $18,00^{c} \pm 3,63$ & 598 & $19,97^{b} \pm 2,20$ & 1218 & $22,94^{\mathrm{a}} \pm 2,25$ & 157 & $22,34^{a} \pm 2,77$ \\
\hline $\mathrm{LP}(\mathrm{mm})$ & 6 & $9,67^{c} \pm 3,50$ & 598 & $11,53^{b} \pm 2,17$ & 1218 & $13,42^{a} \pm 2,27$ & 157 & $12,87^{a} \pm 2,12$ \\
\hline Alt $(\mathrm{mm})$ & 6 & $12,17^{c} \pm 2,32$ & 598 & $13,47^{b} \pm 1,35$ & 1218 & $15,03^{\mathrm{a}} \pm 1,28$ & 157 & $15,30^{\mathrm{a}} \pm 1,62$ \\
\hline
\end{tabular}

CC: Comprimento da carapaça; LC: Largura da carapaça; CP: Comprimento do plastrão; LP: Largura do plastrão e Alt: altura dos filhotes.

Peso 1 = Menos que $2 \mathrm{~g}$; Peso 2 = 2,1 a $4 \mathrm{~g}$; Peso $3=4,1$ a $6 \mathrm{~g}$; Peso 4 = Maior que $6 \mathrm{~g}$.

$\mathrm{a}, \mathrm{b}, \mathrm{c}$ : Letras diferentes nas linhas indicam diferença estatística pelo teste Duncan ( $p \leq 0,001)$; letras iguais não diferem entre si significativamente.

Tabela VI. Coeficiente de correlação de Pearson entre o peso e as primeiras medidas morfométricas de 1979 filhotes de muçuãs (Kinosternon scorpioides) recém-eclodidos (Pearson correlation coefficient between the weight and the first morphometric measurements of 1979 muçuãs (Kinosternon scorpioides) hatchlings).

\begin{tabular}{|c|c|c|c|c|c|c|}
\hline & $P(g)$ & $\mathrm{CC}(\mathrm{mm})$ & $\mathrm{LC}(\mathrm{mm})$ & $\mathrm{CP}(\mathrm{mm})$ & $\mathrm{LP}(\mathrm{mm})$ & $\mathrm{ALT}(\mathrm{mm})$ \\
\hline$P(g)$ & 1,00000 & & & & & \\
\hline $\mathrm{CC}(\mathrm{mm})$ & $\begin{array}{l}0,51917 \\
<0,0001\end{array}$ & 1,00000 & & & & \\
\hline $\mathrm{LC}(\mathrm{mm})$ & $\begin{array}{l}0,36692 \\
<0,0001\end{array}$ & $\begin{array}{l}0,56795 \\
<0,0001\end{array}$ & 1,00000 & & & \\
\hline $\mathrm{CP}(\mathrm{mm})$ & $\begin{array}{l}0,42503 \\
<0,0001\end{array}$ & $\begin{array}{l}0,71192 \\
<0,0001\end{array}$ & $\begin{array}{l}0,49156 \\
<0,0001\end{array}$ & 1,00000 & & \\
\hline $\mathrm{LP}(\mathrm{mm})$ & $\begin{array}{l}0,29198 \\
<0,0001\end{array}$ & $\begin{array}{l}0,55921 \\
<0,0001\end{array}$ & $\begin{array}{l}0,48618 \\
<0,0001\end{array}$ & $\begin{array}{l}0,59563 \\
<0,0001\end{array}$ & 1,00000 & \\
\hline $\operatorname{ALT}(\mathrm{mm})$ & $\begin{array}{l}0,45358 \\
<0,0001\end{array}$ & $\begin{array}{l}0,51429 \\
<0,0001\end{array}$ & $\begin{array}{l}0,44728 \\
<0,0001\end{array}$ & $\begin{array}{l}0,44836 \\
<0,0001\end{array}$ & $\begin{array}{l}0,40274 \\
<0,0001\end{array}$ & 1,00000 \\
\hline
\end{tabular}

P: Peso; CC: Comprimento da carapaça; LC: Largura da carapaça; CP: Comprimento do plastrão; LP: Largura do plastrão e Alt: Altura dos filhotes.

não diferiram estatisticamente entre si. Isto sugere que o ganho em peso se associa ao ganho de massa corpórea e não, necessariamente, ao crescimento ósseo corporal dos filhotes, por um determinado período.

Visando estudar o grau de associação entre as variáveis, peso e medidas corporais dos filhotes, utilizou-se o coeficiente de correlação de Pearson conforme consta na tabela VI. As correlações das características biométricas com o peso dos filhotes demonstraram que as correlações encontradas são positivas $(p \leq 0,0001)$ entre todas as variáveis. Observou-se maior correlação entre as variáveis de comprimento da carapaça (CÇ) com o comprimento do plastrão $(\mathrm{CP})$, e entre o comprimento e a largura do plastrão (CP e LP), correlações mais baixas foram encontradas entre peso $(\mathrm{P})$ e largura do plastrão (LP). As outras características tiveram correlação mediana entre si, demonstrando que correlações entre as medidas corporais são mais fortes, quando associadas entre si do que com o peso, nessa fase inicial de crescimento.

\section{CONCLUSÕES}

A espécie apresentou comportamento reprodutivo sazonal, sugerindo que a influência de fatores ambientais que, se controlados, podem ser minimizados. A relação macho:fêmea de animais em reprodução, observada para cativeiro foi 1:10. A viabilidade dos ovos é um dos principais gargalos do sistema produtivo de muçuãs em cativeiro, uma vez que apenas $20 \%$, em média, dos ovos postos eclodiram no período estudado. Na fase de crescimento as características corporais não sofrem a influência direta do peso. Características como acasalamento, período de incubação e eclosão, com estudos bem definidos, permitem delinear um adequado manejo reprodutivo, para dar suporte aos sistemas de criação em cativeiro para a espécie.

\section{BIBLIOGRAFIA}

Araújo, J. da C. 2009. Parâmetros produtivos e qualidade de ovos de muçuãs (Kinosternon scorpioides) submetidos a manejo alimentar diferenciado. Dissertação (Mestrado em Zootecnia). Universidade Federal de Lavras. Minas Gerais. $111 \mathrm{f}$.

Barreto, L.; Lima, L.C. e Barbosa, S. 2009. Observations on the ecology of Trachemys adiutrix and Kinosternon scorpioides on Curupu Island, Brazil. Herp Rev, 40: 283-286.

Basho, A.Y. e Molina, F.B. 2000. Conservação ex situ de quelônios neotropicais: Biologia reprodutiva do muçuã, Kinosternon scorpioides scorpioides (reptilia, Testudines, kinosternidae). Arq InstBiol, 67: 1-145. 
Cagle, F.R. 1950. The life history of the slider turtle. Pseudemys scripta troostii. Ecol Monog, 1: 31-54.

Carpenter, C.C. 1980. An ethological approach to reproductive sucess in reptiles. In: Murphy, J.B. and Collins, J.T. (Eds.). Reproductive biology and diseases of captive reptiles. Society for the Study of Amphibians and Reptiles. Kansas. pp 33-48

Carpenter, C.C.; Ferguson, G.W. 1997. Variation and evolution of stereotyped behavior in reptiles. In: Gans, C.; Tinkle, D.W. Biology of reptilia: Ecology and behavior. Academic Press. London, UK. pp. 335-554.

Carvalho, R.C.; Oliveira, S.C.R.; Bombonato, P.P.; Oliveira, A.S. e Sousa, A.L. 2010. Morfologia dos órgãos genitais masculinos do Jurará Kinosternon scorpioides (Chelonia: Kinosternidae). Pes Vet Bras, 30: 289-294.

Castro, A.B. 2006a. Biologia reprodutiva e crescimento do muçuã Kinosternon scorpioides (Linnaeus, 1776) em cativeiro. Dissertação (Mestrado em Ciência Animal). Universidade Federal do Pará. Belém-PA. $101 \mathrm{f}$.

Castro, A.B. 2006b. Dimorfismo sexual em muçuã)/Kinosternon scorpioides). Congresso internacional sobre manejo de fauna silvestre na Amazônia e América Latina, Ilhéus. Anais... UESC. Ilhéus.

Clayton, L. e Milner-gulland, J. 2000. The trade in wildlife in North Sulawesi, Indonesia. In: Robinson, J.G.; Bennett, E.L. Hunting for sustainability in tropical forests. Columbia University Press. New York. 24: 473-496.

Costa, F.B.; Alves, F.R.; Costa, A.P.; Barros, A.C.E.; Guerra, P.C.; Sousa, A.Le Oliveira, A.S. 2009. Ultrasonographic and radiographic determination of egg development of jurarás (Kinosternon scorpioides) in aptivity. Pes Vet Bras, 20: 841-846.

Ferri, V. 2002. Turtles e tortoises: A Firefly guide. Firefly Books. 256 pp.

Gibbons, J.W. 1970. Reproductive dynamics of a turtle (Pseudemys scripta) population in a reservoir receiving heated effluent from a nuclear reactor. Canad J Zool, 48: 881-885.

IBAMA. Instituto Brasileiro do Meio Ambiente e dos Recursos Naturais Renováveis. Lei n. 5.197, de 03 de janeiro de 1967. Dispõe sobre a proteção à fauna e dá outras providências. In: Legislação de Conservação da Natureza, $4^{a}$ ed. rev. e atual. F.B.C.N. São Paulo. pp.132-138.

IBAMA. Instituto Brasileiro do Meio Ambiente e dos Recursos Naturais Renováveis. Lei n. 9.605, de 12 de fevereiro de 1998. Dispõe sobre as sanções penais e administrativas derivadas de condutas e atividades lesivas ao meio ambiente, e dá outras providências (12/09/2015).

Iverson, J.B. 1991. Life history and demography of the yellow mud turtle, Kinosternon flavescens. Herpetologica, 47: 373-395.

Kirkpatrick, D.T. 1997. The biology, husbandry and health care of reptiles. In: L. Ackerman. The husbandry of reptiles: Volume II. T.F.H Publications. Neptune, New Jersey. pp. 447-463.

Koeppen, W. 1948. Climatología. México. Fondo de cultura económica. $478 \mathrm{pp}$.

Marques, J.R.F.; Costa, M.R.; Camargo JR,R.N.C.; Albuquerque, M.S.M.; Marques, L.C. e Aguiar, J.F. 2008. Conservação e melhoramento dos recursos genéticos animais da Amazônia brasileira. In: $X$ Congresso internacional de zootecnia, ZOOTEC/2008. João Pessoa-PB. Anais... Perfil profissional e demanda de mercado. João Pessoa-PB: ABZ UFPB/CCA. Embrapa caprinos, 1: 1-14.

Molina, F.B. 1989. Observações sobre a biologia e comportamento de Phrynops geofiroanus em cativeiro (Reptilia, Testudines, Chelidae). Dissertação (Mestrado em Zoologia). Departamento de Zoologia do Instituto de Biociência. Universidade de São Paulo. São Paulo-SP.
Molina, F.B. 1990. Reproductive biology of Phrynops geoffroanus (Testudines: Chelidae) in captivity. Tortoise e Turtles, 5: 8.

Moll, E.O. e Legler, J.M. 1971. The life history of a Neotropical slider turtle, Pseudemys scripta (Shoepff), in Parama. Bulletin of the Los Angeles County. Museum of Natural History. Science, 11. 102 pp.

Mondolfi, E. 1955. Anotaciones sobre la biologia de três quelônios de los llanos de Venezuela. Memoria de La Sociedad de Ciencias Naturales La Salle, Caracas, 15: 177-183.

Pritchard, P.C.H. 1979. Encyclopedia of Turtles. T.F.H. Publications. Neptune, New Jersey. 859 pp.

Redford, K.H. 1997. A floresta vazia. In: Valladares-Padua, C., Bodmer, R.E., Cullen Júnior, L. Manejo e conservação de vida silvestre no Brasil. Sociedade Civil Mamirauá. CNPq/Belém. Brasília. 1: 01-22.

Rocha, M.B. e Molina, F.B. 1990. Reproductive biology of Kinosternon scorpioides (Testudines: Kinosternidae) in captivity. Tortoises e Turtles, 5: 8-8.

Rueda-Almonacid, J.V.; Carr, J.L.; Mittermeier, R.A. Rodríguez-Mahecha, J.V.; Mast, R.B.; Vogt, R.C.; Rhodin, A.G.J.; Ossa-Velásquez, J. De La; Rueda, J.N. e Mittermeier, C.G. 2007. Las tortugas y los cocodrilianos de lós países andinos del trópico. Serie de guias tropicales de campo $N^{\circ}$ 6. Conservación Internacional. Editorial Panamericana. Formas e Impresos. Bogotá, Colômbia. 538 pp.

Sampaio, P.A.M. 2003. Comércio ilegal de carne de animais silvestres em quatro feiras livres do estuário amazônico, Estado do Pará - Brasil. Trabalho de Conclusão de Curso (Bacharelado em Ciências Biológicas). Universidade Federal do Pará. Belém-Pará.

SAS. 2004. Institute Inc. SAS/ STAT ${ }^{\circledR} 9.0$ User's Guide. SAS Institute Inc. Cary, NC.

Schneider, L.; Ferrara, C. e Vogt, R.C. 2010. Description of behavioral patterns of Podocnemis erythrocephala (Spix, 1824) (Testudines: Podocnemididae) (Red-headed river turtle) in captivity, Manaus, Amazonas, Brazil. Act Amaz, 40: 763-770.

Silva, A.S.L. 2006. Aspectos biológicos e econômicos da criação de muçuã (Kinosternon scorpioides, Linnaeus, 1766) em cativeiro. Trabalho de Conclusão de Curso (Bacharelado em Medicina Veterinária). Universidade Federal Rural da Amazônia, Belém. Pará.

Silva, A.S.L. 2011. Aspectos reprodutivos do muçuã (Kinosternon scorpioides) em cativeiro. Dissertação (Mestrado em Medicina Veterinária). Faculdade de Ciências Agrárias e Veterinárias. Universidade Estadual Paulista. Jaboticabal- SP.

Sirvinskas, L.P. 2002. Direito ambiental, fauna, tráfico e extinção de animais silvestres. Rev Jur, 50: 298.

Souza, F.L. 2004. Uma revisão sobre padrão de atividade, reprodução e alimentação de cágados brasileiros (Testudines: Chelidae). Phyllomedusa, 3: 15-27.

Tinkle, D.W.; Congdon, J.D. e Rosen, P.C. 1981. Nesting frequency and success: implications for the demography of painted turtles. Ecology, 62: 1426-1432.

Traffic. 2005. Wildlife trade: what is it? http://www.traffic.org/trade (17/09/2015).

Vogt, R.C. 2008. Amazon Turtles. Gáfica Biblos. Lima. 104 pp. 